ston et al. 1973) so that if it is reduction in gastric secretion which permits healing of mucosal ulcers a therapeutically satisfactory pharmacological agent would presumably have to reduce acid secretion by an equal amount, particularly during the intendigestive periods of the day and during the night. The results of the present study confirm the findings of Wyllie et al. (1973) in healthy volunteers and indicate that in patients with duodenal ulcer Metiamide is capable of reducing gastric secretion to levels seen after vagotomy. A therapeutic trial of Metiamide in the treatment of duodenal and gastric ulceration (and related conditions such as hiatus hernia) is therefore warranted.
K. G. W. is in receipt of a grant from the Scottish Hospital Endowments Research Trust. The Metiamide was kindly supplied by Dr. M. Bloch, Smith, Kline and French Laboratories Ltd., Welwyn Garden City.

Requests for reprints should be sent to Dr. K. G. Wormsley, Ninewells Hospital, Dundee DD2 1UB.

\section{References}

Hunt, J. N. (1948). Biochemical fournal, 42, 104.

Johnston, D., et al. (1973). Gastroenterology, 64, 1 Wyllie, J. H., Ealding, W. D. P., Hesselbo, T., and Black, J. W. (1973).
Gut, 14, 424.

\title{
Delayed Postpartum Sterilization
}

\section{J. MOWAT}

British Medical fournal, 1974, 2, 306-308

\section{Summary}

A series of 75 women who requested immediate postpartum sterilization were told this would not be carried out till three months postpartum, and only 64 women subsequently underwent sterilization. While some may regard this as a failure of a family limitation programme, the mental well-being of the woman is better served by allowing time for reflection before undergoing what is virtually an irreversible procedure.

Though depot medroxyprogesterone acetate was associated with irregular and unpredictable bleeding in almost $25 \%$ of the patients its acceptability and value as a temporary postpartum contraceptive was confirmed.

\section{Introduction}

Limitation of family size by immediate postpartum sterilization is now common practice. Some gynaecologists, however, have reservations about whether pregnancy and the very early puerperium is the correct time to consider seriously or perform such an irreversible and permanent procedure, particularly when these operations are today being carried out in younger patients with a lower parity then ever before.

In the present investigation all patients who during pregnancy requested sterilization were informed that this would be carried out 12 weeks postpartum, if they still wished it, and not immediately after delivery. Since there was a possibility of a pregnancy occurring before sterilization was performed the patients were offered an injection of medroxyprogesterone acetate (Depo-Provera) for contraceptive pumposes. The questions comsidered in this study were, firstly, the number of patients subsequently reconsidering their original request for sterilization and, secondly, the acceptability of medroxypnogesterone as a temporary postpantum contraceptive.

\section{Patients and Methods}

All patients attending the antenatal clinics of one unit in the Glasgow Royal Maternity Hospital who requested sterilization

Victoria Infirmary, Glasgow G42 9TY

J. MOWAT, M.R.C.O.G., F.R.C.s., Consultant Obstetrician and Gynaecologist were included in the present series. Over a period of twelve months during which there were 1,267 deliveries in the unit, 75 patients in their antenatal period asked for sterilization. The patients were told that sterilization would be carried out between eight and 12 weeks postpartum and not in the period immediately after delivery. After delivery they were offered an injection of $150 \mathrm{mg}$ intramuscular medroxyprogesterone acetate as a contraceptive, and all accepted. The medroxyprogesterone was given on the fifth or sixth day after delivery. An appointment was made for postnatal examination at six weeks, when a convenient time for admission for sterilization was arranged. Four patients were sterilized by Pomeroy tubal ligation and the remainder at laparoscopy. Laparoscopy was undertaken as described by Steptoe (1967) and sterilization performed by tubal coagulation, without division of the tubes (Mowat, 1974). The patients stayed in hospital for two nights after laparoscopy. They were asked to return for reviow at three months and thereafter as necessary. At these follow up appointments a careful note was made of any bleeding irregularities, vaginal discharge, or any other side effects which the patient may have noticed.

\section{Results}

The average age of the patients was 32 years and the average parity was five (tables I and II).

TABle I-Age Groups of 75 Patients who requested Postpartum Sterilization

\begin{tabular}{cc|c|c|c|c|c}
\hline Age . of patients & $\because$ & $20-24$ & $\begin{array}{c}25-29 \\
\text { No. of }\end{array}$ & $\begin{array}{c}30-34 \\
26\end{array}$ & $\begin{array}{c}35-39 \\
19\end{array}$ & $\begin{array}{c}40 \\
8\end{array}$ \\
\hline
\end{tabular}

TABLE II-Parity of 75 Patients who requested Postpartum Sterilization

\begin{tabular}{|l|l|l|l|l|l|l|l|l|l|l|l|}
\hline Parity & 2 & 2 & 3 & 4 & 5 & 6 & 7 & 8 & 9 & 10 & 11 \\
\hline
\end{tabular}

Seventy-five patients requested sterilization while still pregnant, of whom three did not return for postnatal examination, and attempts to trace them by the health visitor were unsuccessful. Eight patients said they no longer wanted sterilization because they considered that it was too drastic a form of contraception. All were on the pill. Whether any of these patients will become pregnant in the future remains to be seen 
but one patient returned requesting that sterilization be performed. No patient who refused sterilization would agree on direct questioning that readmission to hospital was a problem either because of difficulties in looking after the new baby or for any other social or domestic reason.

Sixty-four patients were sterilized, and after at least three months, though early for proper assessment, no one expressed any regrets. Twenty-eight patients did not return for followup after their operation, and information about them was obtained from their general practitioners.

Fifty-six patients were sterilized before 14 weeks postpartum. Eight were sterilized after this time, two of whom showed the presence of a corpus luteum at the time of their operation, inciicating that ovulation had probably occurred, with the consequent danger of pregnancy.

Vaginal staining for three to four weeks postpartum was very common, and nine patients still had irregular staining at the six-week postnatal visit. One patient was readmitted to hospital 10 days after delivery and evacuation of the uterus for retained products of conception was performed.

Twenty-nine patients did not suffer any abnormal bleeding and all began menstruating normally 14-16 weeks after delivery apart from two patients who did not menstruate for periods of 12 and 18 months postpartum. Most complaints of irregular bleeding occurred between eight and 16 weeks after delivery though some continued longer than this. Though in most cases the bleeding reported was intermittent staining 19 patients complained of irregular heavy bleeding. Of these 19 , 10 did not seek advice for treatment. Nine patients went to their general practitioner; five were put on the "pill" to regulate their bleeding and four were referred to hospital and had diagnostic curettage performed, but in only one was endometrium obtained and this was reported as showing "chronic endometritis." Three patients said that they were unable to have intercourse because of the heavy bleeding. Only two patients, however, admitted that they would have preferred not to have had the injection because of the bleeding. All the other patients who were followed up said they were relieved to have had the injection and to know that they were safe from pregnancy for at least three months after delivery.

All but four patients had an endometrial biopsy performed before undergoing sterilization. The most common findings were a progestational endometrium (in 38 patients) and inactive endometrium (in 12 patients) though in 10 patients no curettings were obtained at all.

Only one patient in the series breast-fed her baby and the medroxyprogesterone did not apparently interfere with lactation.

\section{Discussion}

An important side effect after sterilization is patient regret. Black and Sclare (1968) found that $3.6 \%$ of their patients later regretted the operation. Any patient who has a sterilization operation may later regret the finality of it. It was felt, therefore, that a decision taken in the emotional atmosphere of a pregnancy, particularly if unplanned, may more often be repented at a later date. If these patients could consider the problem in an unhurried way, however, with no pressure on them the proportion who later have second thoughts may diminish.

In this series of 75 patients voluntarily requesting sterilization during their pregnancy 11 later changed their minds though one subsequently again decided that she would like to be sterilized. There was no evidence if all the patients had been sterilized immediately postpartum that any would later have had regrets, but perhaps the patient's individual well-being has benefitted. Adams (1964) has advocated a "cooling off" period before performing sterilization, and this has been reiterated more recently by Emens et al. (1973).
One of the problems which patients may have is their inability to arrange readmission to hospital because of the young baby or for some other social or domestic reason. No patient in this series admitted that readmission to hospital was a drawback. Laparoscopy has also been of great benefit in this respect because of the short postoperative stay necessary. Though the patients in this series stayed in hospital for two nights after the laparoscopy we are now happy to discharge patients the day after. Many gynaecologists perform laparoscopy as an outpatient procedure under general or local anaesthesia (Chaturachinda, 1972), and the incidence of complications and the failure rate are low (Liston et al., 1970; Mowat, 1974).

A serious problem facing the patient who has an immediate postpartum sterilization is the small but real risk of her baby-apparently quite healthy at binth and in the first few days of life-dying shortly after as the result of some undetected congenital abnormality. Though such a loss may be slightly less upsetting to the very highly parous woman it could be a catastrophe for the woman who has been sterilized after having only one or two other children. There may also be a medical contraindication in some patients-for example, heart disease-to performing immediate postpartum sterilization. For these reasons the use of medroxyprogesterone as a temporary postpartum contraceptive may be valuable.

Disorganization of the menstrual cycle has been noted as the most common side effect of treatment with medroxyprogesterone acetate (Jeppson, 1972; Powell and Seymour, 1971). It was hoped that if it were given as a once-only immediate postpartum injection, this would not be a problem. Jones and Lonky (1971) noted a high incidence of spotting immediately postpartum with the use of medroxyprogesterone acetate, but with a gradually diminishing severity. Karim $e t$ al. (1971) considered that amenorrhoea was the most important side effect of the postpartum use of medroxyprogesterone, while El-Mahgoub et al. (1972) were worried about the prolonged and unpredictable intervals of resumption of spontaneous ovulation after discontinuing therapy. In patients who are to be sterilized, however, neither amenorrhoea nor subsequent fertility is a matter of importance. Irregular and heavy episodes of bleeding, on the other hand, may be a source of great distress and discomfort to the patient. In spite of this abnormal bleeding, which was considerable in 19 of the patients in this series, most patients (69 out of 72) were happy to have had the long-term contraceptive, and it can be recommended as a suitable temporary contraceptive in the postpartum period.

Irregularity of bleeding could be satisfactorily controlled by the use of a combined oestrogen and progestogen mixture. There was no correlation between the presence or absence of irregular bleeding and the state of the endometrium, which in the presence of a history of abnormal episodes of spotting or bleeding could be inactive, show a progestational effect, or be absent altogether. Three of the four patients who were admitted as emergencies because of heavy vaginal bleeding and on whom diagnostic curettage was performed had no endometrium. It was interesting to record that many of the patients who complained of heavy bleeding considered that it was related to exertion in one form or other. Apart from the irregular episodes of bleeding, unpredictable in time and amount, no other side effect was noted.

I should like to thank Dr. M. M. Garrey and Dr. A. W. Laughland for allowing me to study their patients, and Mrs. Morris, health visitor at Glasgow Royal Maternity Hospital for her help in the follow-up of the patients.

\section{References}

Adams, T. W. (1964). American fournal of Obstetrics and Gynecology, 89, 395. Black, W. P., and Sclare, A. B. (1968). Fournal of Obstetrics and Gynaecology of the British Commonwealth, 75, 219. 
Chaturachinda, K. (1972). American fournal of Obstetrics and Gynecology,

El-Mahgoub, S., Karim, M., and Ammar, R. (1972). Acta Obstetrica and Gynecologica Scandinavica, 51, 251.

Emens, J. M., Jordan, J. A., and McLaren, H. C. (1973). British Medical fournal, $2,421$.

Jeppson, S. (1972). Acta Obs.etricia and Gynecologica Scandinavica, 51, 257. Jones, J. R., and Lonky, S. (1971). New York State Journal of Medicine, 71,
2279.
Karim, M., et al. (1971). British Medical fournal, 1, 200.

Liston, W. A., Bradford, W. P., Downie, J., and Kerr, M. G. (1970). Lancet, 1, 382.

Mowat, J. (1974). Ob-Gyn Digest. In press.

Mowat, J. (1974). Ob-Gyn Digest. In press.
Powell, L. S., and Seymour, R. J. (1971). American fournal of Obstetrics and Gynecology, 110, 36.

Steptoe, P. C. (1967). Laparoscopy in Gynaecology. Edinburgh, Livingstone.

\title{
Surgical Reduction of Tumour Bulk in Management of Abdominal Burkitt's Lymphoma
}

\author{
I. T. MAGRATH， S. LWANGA，W. CARSWELL， N. HARRISON
}

British Medical fournal, 1974, 2, 308-312

\section{Summary}

The results of a retrospective study of the value of reductive surgery in the treatment of abdominal Burkitt's lymphoma are reported. Nine patients had almost complete removal of the tumour, 16 had little over half of the tumour removed, and 43 underwent biopsy only. All patients subsequently received chemotherapy. There was a highly significant difference in the proportion of patients achieving a sustained durable remission $(P>0.0001)$ and a significant difference in survival $(P>0.05)$ between the group having almost complete removal and the partial resection group. Partial resection gave no advantage over no surgery. We believe that whenever possible complete removal of the abdominal tumour should be attempted regardless of the presence of extra-abdominal tumour; but unless at least $90 \%$ of the tumour can be removed there is no advantage in partial resection in terms of response to subsequent chemotherapy. In particular the removal of one of two involved ovaries can no longer be recommended. The implications of these results are related to cancer treatment strategy.

\section{Introduction}

Burkitt's lymphoma is an exceptional tumour in many respects, not least of which is its dramatic response to chemotherapy. Some $80 \%$ of all patients admitted to the Lymphoma Treatment Centre, Kampala, achieve complete clinical remission after chemotherapy (Ziegler, 1972), and though about twothirds of these relapse the overall predioted long-term survival rate based on 185 patients treated at the centre between 1967 and 1972 is 57\% (Magrath and Ziegler, 1973). The relapse and survival rates depend largely on whether abdominal tumour is present. Where there is only extraabdominal tumour, regardless of site, cumulative long-term

\section{Lymphoma Treatment Centre, Kampala, Uganda}

I. T. MAGRATH, M.B., M.R.C.P., Director (Present address: Department of Pediatric Oncology, National Cancer Institute, Bethesda, Maryland 20014, U.S.A.)

Makerere University, Kampala, Uganda

S. LWANGA, B.sc., Medical Statistician

Mulago Hospital, Kampala, Uganda

W. CARSWELL, M.B., F.R.C.S., Senior Surgical Registra

N. HARRISON, M.D., F.R.C.S., Senior Surgical Registrar (Present address: Institute of Urology, St. Peter's and St. Paul's Hospitals, London) survival is $82 \%$, whereas with abdominal tumour (not extensively resected surgically) it ranges from $29 \%$ to $52 \%$ depending on the presence and site of extra-abdominal tumour (table I). This finding raises the question whether complete or nearly complete resection of abdominal tumour, when possible, would improve the response to chemotherapy in terms of relapse and survival. Since $39 \%$ of male patients and $73 \%$ of female patients with Burkitt's lymphoma have abdominal involvement, and since ovarian tumour, which is readily resected, accounts for over half of the abdominal tumours in female patients (Magrath, 1974 a), this is an important consideration.

TABLE I-Clinical Staging of Burkitt's Lymphoma and Relation to Prognosis. Results were Calculated Actuarially (see Text)

\begin{tabular}{l|c|c|c}
\hline Stage A & Extent of Tumour & $\begin{array}{c}\text { Survival Rate } \\
(100 \text { Weeks or } \\
\text { More) }\end{array}$ & $\begin{array}{c}\text { Remission Rate* } \\
\text { (50 Weeks or } \\
\text { More) }\end{array}$ \\
\hline Stage B & $\begin{array}{c}\text { Single tumour } \\
\text { outside } \\
\text { abdomen } \\
\text { Multiple extra- } \\
\text { abdominal } \\
\text { tumours (inclu- } \\
\text { ding nervous } \\
\text { system) } \\
\text { Abdominal } \\
\text { tumour with } \\
\text { or without facial } \\
\text { tumour } \\
\text { Abdominal } \\
\text { tumour with } \\
\text { tumour at other } \\
\text { sites (except } \\
\text { face alone) }\end{array}$ & $82 \%$ & $61 \%$ \\
\hline
\end{tabular}

*Remission rate represents proportion of patients who have not relapsed.

Previously chemotherapy has been used as an adjunot to surgery, putatively to prevent the development of metastases (Nora and Preston, 1963; Fisher, 1972). This mode of utilization, however, has met with little success (Wolff et al., 1968; Fisher, 1972), and in multicentric tumours or tumours which are potentially widely disemminated from the outset-for example, the lymphomas-a radically different approach has been necessary. In these cases there has been a reversal of roles, with surgery in effect being used as a relatively minor adjunct to chemotherapy. In the treatment of abdominal Burkitt's lymphoma surgery has possibly a much more important place, and in this paper we attempt to evaluate the potential advantages of a combined surgical and chemotherapeutic approach and in particular to determine the importance of the extent of surgical resection of tumour bulk. Since radiotherapy is unavailable in Uganda it played no part in the treatment of these patients. 